\title{
Medicina alternativa: intento de análisis
}

\author{
Adolfo Peña ${ }^{1}$, Ofelia Paco ${ }^{2}$
}

"We should forever keep an open mind, but, not so open that our brain falls out" Petr Skrabanek.

Resumen

Palabras clave
Por medicina alternativa se considera al conjunto de disciplinas terapéuticasy diagnósticas que existen fuera de las instituciones del sistema de salud convencional. El uso actual de esta 'clase' de medicina está muy extendido, tanto en el mundo industrial como el preindustrial. Parte del creciente uso de las terapias alternativas se debe a su reciente validación profesional; muchos textos de divulgación general claman y justifican su uso, basándose en información académica nonecesariamente de rigor científico. En este ensayo, se analizará la evidencia científica disponible, así como también se analizará las bases lógicas y filosóficas de la medicina en cuestión.

Medicina alternativa; terapias alternativas; acupuntura.

\begin{abstract}
Alternative medicine: Intent of analysis Abstract

Alternative medicine is considered a set of therapeutic and diagnostic disciplines that exist outside the conventional health system. Current use of this 'class' of medicine is amply extended in both developed and undeveloped world. One of the causes of increasing use of alternative medicine is recent professional validation. Many general divulgation texts claim and justify its use based on academic information without scientific quality. In this essay we will analyze the available scientific evidence as well as logical and philosophical bases of this so called medicine.
\end{abstract}

Key words: Alternative medicine; alternative therapies; acupuncture.

\section{Introducción}

La 'medicina occidental' ha padecido diferentes cambios a través de los tiempos. En sus primeros años, la filosofía naturalista de los jonios presocráticos fue su rectora; la influencia teleológica aristotélica tomó

1 Residente Senior de Medicina Interna, Good Samaritan Hospital. Cincinnati, Ohio, USA.

2 Servicio y Manejo de Datos, PPD Global Central Labs. Highland Heights, Kentucky, USA. luego la posta $\mathrm{y}$, a mediados de nuestra era, los vitalistas la dominaron $\left({ }^{1}\right)$. La medicina, como la conocemos hoy, es pues de aparición reciente, quizá de apenas dos siglos.

La revolución científica liderada por Galileo, en el siglo XVII, introdujo cambios inconmensurables, tanto en la esfera intelectual como social. La medicina pronto sufrió los embates del pensamiento científico y poco a poco reconfiguró sus postulados y sus métodos de conocimiento. Así, en uno de los eventos considerado clave en la reforma de la profesión médica, la publicación del informe Flexner $\left({ }^{2}\right)$, en 1910, se estableció como objetivo clave hacer de la medicina una profesión científica $\left(^{3}\right)$.

Sin embargo, en el curso de las últimas décadas, han reaparecido en el sistema de salud 'nuevas' terapias. En aras de la apertura mental y la tolerancia, la mal llamada 'medicina occidental' está tratando de ser 'integrada' con disciplinas antes llamadas alternativas y hoy complementarias.

El uso de la medicina alternativa hoy en día está muy extendido; ya no es patrimonio de sociedades con historia cultural tra- 
dicional, como la nuestra. Por ejemplo, en una encuesta realizada en los Estados Unidos, un tercio de los encuestados refirió haber usado al menos una terapia no convencional en un periodo de doce meses $\left({ }^{4}\right)$. Asimismo, se ha calculado que el número de visitas a establecimientos de medicina alternativa, en los Estados Unidos, asciende a 425 millones, cifra que supera al número de visitas a los consultorios médicos convencionales (388 millones) $\left({ }^{4}\right)$.

Es de señalar que la investigación científica también está dedicando recursos a la medicina alternativa; y algunos resultados le son favorables; por ejemplo, dos metaanálisis recientes refieren que algunos efectos terapéuticos de la homeopatía parecen no ser atribuibles al efecto placebo $\left({ }^{5}\right)$.

El lugar de la medicina alternativa en el sistema de salud no es, pues, marginal; por ello, es imperativo su estudio y análisis. Varias opiniones han sido vertidas frente al desarrollo creciente de la medicina alternativa. Algunos opinan que no debemos confundir la charlatanería con la genuina medicina tradicional y alternativa, la cual valdría por sí misma sin necesidad de clamar a la ciencia $\left({ }^{6}\right)$. Otros son de opinión que el perjuicio científico positivista no debe ocluir nuestra tolerancia, negando 'genuinas' posibilidades terapéuticas. Hay quienes creen que la medicina alternativa puede ser la solución frente al creciente encarecimiento de la medicina occidental, hecho más crítico en los países pobres, como el nuestro. En fin, el debate está abierto y, al juzgar por el volumen de publicaciones, la tolerancia parece ser la vencedora.

En la actualidad, se suele invocar pues a la tolerancia y a la 'mentalidad abierta' a fin de no caer preso de la ciencia y así incurrir en el prejuicio. Empero, creo que el prejuicio es resultado de una postura emocional; equivale al rechazo apriorístico antes de haber examinado las pruebas que pretenden sustentar una opinión. Si debemos determinar la veracidad de un asunto, debemos abordarlo con una apertura mental tan grande como sea posible, pero también debemos hacerlo con la bondad ofrecida por la crítica. Si tras un análisis cuidadoso rechazamos una proposición determinada, no incurriríamos en prejuicio; sino tal vez en 'posjuicio' (juicio a posteriori).

\section{¿Medicina alternativa, complementaria o tradicional?}

Uno de los primeros problemas que nos enfrentamos al analizar la medicina alternativa es el cómo definirla. Así lo demuestra la variedad de propuestas aparecidas en prestigiosas publicaciones. Según autores independientes, la medicina complementaria se la define como el grupo de disciplinas terapéuticas y diagnósticas que existen fuera de las instituciones donde el sistema de salud convencional es brindado o enseñado $\left({ }^{4,7}\right)$.

La colaboración Cochrane, insigne propulsora del 'movimiento' medicina basada en evidencias, ha adoptado una definición semejante: se entiende por medicina complementaria a todas aquellas prácticas y recursos de curación acompañadas de sus respectivas teorías y creencias, que no son intrínsecas al sistema de salud políticamente dominante de una sociedad o cultura en un periodo histórico determinado $\left(^{8}\right)$.

Para la Organización Mundial de Salud, el uso de términos como 'medicina alternativa' y 'medicina complementaria' responde a una clasificación otorgada en países donde no existe integración entre medicina tradicional y medicina occidental (pp. $\left.{ }^{1}\right)\left({ }^{9}\right)$. Por ello, la OMS prefiere hablar en 'términos' de medicina tradicional. Según la OMS, aquel vocablo debe referirse tanto a los sistemas de medicina tradicional -por ejemplo, la medicina tradicional china, el ayurveda hindú, y la medicina unani árabe-, así como a las diversas formas de medicina indígena no sistémicas $\left({ }^{\text {pp. }}{ }^{1}\right)\left({ }^{9}\right)$. 
Algunos se han sentido disconformes con estas propuestas, señalando que no es cierto que la medicina alternativa se encuentre totalmente fuera del sistema convencional $\left({ }^{10}\right)$. En efecto, algunas escuelas de medicina, hospitales y otros servicios de salud norteamericanos ofrecen medicina no convencional como alternativa terapéutica y de enseñanza $\left({ }^{11,12}\right)$; inclusive, algunos estados norteamericanos ofrecen, en sus coberturas de seguro, coberturas para terapias no convencionales $\left({ }^{13}\right)$. En el Perú, algunas escuelas de medicina han sido anfitrionas de congresos de medicina tradicional $\left({ }^{14}\right)$; el seguro social (EsSalud) posee un programa completo de medicina alternativa $\left({ }^{15}\right)$.

Empero, las propuestas de definición que acabamos de ver entienden por medicina solo un 'constructo' social; su definición es convencional y relativa, determinada por el contexto histórico, tornándose en membrete, no en definición que dé cuenta de la naturaleza ontológica (real) y lógica de lo que 'es' medicina alternativa. Leibovici $\left({ }^{16}\right)$ ha señalado que, usar un rótulo social por definición nos expone a la manipulación y el malentendido; por ejemplo, los medios de comunicación pueden crear un gran impacto si difunden la opinión de que la medicina alternativa es aquella que no pertenece al sistema de salud 'políticamente' dominante.

Se ha señalado, también que, a 'diferencia' de la medicina clásica occidental, muchas de estas disciplinas complementarias no solo claman el alivio de síntomas sino la restauración del bienestar, en un proceso de autocuración enmarcado en una perspectiva 'holística' $\left({ }^{17}\right)$. Algunos han reclamado con cierta razón que tal 'visión holística' no es propiedad de la medicina complementaria, sino propiedad de todo buen clínico $\left({ }^{16}\right)$.

\section{Situación actual de la medicina alternativa}

Como adelantáramos en la introducción, el uso de la medicina complementaria se encuentra bastante difundido. Estudios recientes han determinado que los norteamericanos gastan, por año, aproximadamente 13700 millones de dólares, en servicios de medicina alternativa $\left({ }^{4}\right)$. En lo que respecta a suplementos vitamínicos, se consume alrededor de 2000 millones de dólares. La suma de las cifras anteriores representa casi la mitad de los gastos anuales en servicios médicos de los Estados Unidos $\left({ }^{4}\right)$.

El uso de terapias alternativas en otros países desarrollados también es elevado. Informes indican que un $46 \%$ de los australianos, así como $49 \%$ de franceses y $70 \%$ de canadienses han utilizado alguna de esas terapias $\left({ }^{\text {pp. }}{ }^{11}\right)\left({ }^{9}\right)$. En Alemania, uno de cada tres alemanes ha utilizado alguna terapia alternativa, siendo la acupuntura y la homeopatía las más empleadas $\left({ }^{18}\right)$.

En los países eufemísticamente llamados en 'vías de desarrollo', el uso de la medicina alternativa se considera aún mayor. La OMS parece celebrar que, en estos países, la mayoría de la población siga utilizando la medicina tradicional para satisfacer sus necesidades sanitarias primarias. En uno de sus informes del año 2000 se indica que, en promedio, $80 \%$ de la población africana usó tal medicina; Etiopía es el país con mayor consumo $(90 \%)\left(\right.$ pp. $\left.{ }^{9}\right)\left({ }^{9}\right)$.

En Sudamérica, se sabe que aproximadamente $70 \%$ de la población chilena y $40 \%$ de la población colombiana consumen medicina tradicional $\left({ }^{19}\right)$. En el caso peruano, un estudio ejecutado en un hospital de Lima de cuarto nivel encontró que cerca de $70 \%$ de pacientes utilizó alguna medicina tradicional $\left({ }^{20}\right)$. Una investigación realizada en los años 1980, en barrios marginales de Lima, encontró que $57 \%$ de los encuestados manifestaba una disposición favorable hacia la medicina tradicional $\left({ }^{21}\right)$.

Por otro lado, los gastos en investigación también son importantes. Hace pocos años, el Instituto Nacional de Salud Norte- 
americano (NIH) $\left({ }^{22}\right)$ creó una oficina dedicada a la investigación de la llamada medicina complementaria; su finalidad, explorar sus prácticas curativas en el contexto riguroso de la ciencia, además de educar y entrenar a sus investigadores $\left({ }^{23}\right)$. Aunque su creación produjo cierto malestar entre la comunidad científica norteamericana $\left({ }^{24}\right)$, la oficina ha continuado con sus investigaciones; sus gastos ascendieron de dos millones de dólares, en el año 1993, a sesenta y ocho mil setecientos millones de dólares, en el $2000\left({ }^{25}\right)$. En los países de Europa, también se le dedica dinero a la medicina alternativa; en el Reino Unido, por ejemplo, se invierte aproximadamente $0,08 \%$ del presupuesto nacional de investigación $\left({ }^{26}\right)$.

La herbal medicine ha crecido de forma impresionante. Una encuesta realizada en los EE UU, en 1997, mostró que aproximadamente $12,1 \%$ de los norteamericanos consumió algún producto 'natural'. Esto contrasta con el escaso $2,5 \%$, de $1990\left({ }^{27}\right)$. El mercado mundial de medicinas elaboradas con hierbas basadas en el conocimiento tradicional se estima en la actualidad (según la OMS) en 60 mil millones de dólares $\left({ }^{9}\right)$.

De acuerdo a las cifras referidas previamente, la medicina complementaria, sobretodo en los países industrializados, parece estar lejos de representar una medicina tradicional. Perteneciente a la cultura popular, la medicina tradicional se aprendía, cultivaba y difundía en el hogar y en la comunidad. Rara vez era registrada de manera permanente, de modo que su transmisión quedaba librada a la tradición oral; con pocas excepciones, era anónima. La medicina alternativa actual no es anónima sino, por el contrario, muy publicitada; sus productos son fabricados en masa para el gran público; su afán no parece ser solo el de curar, aliviar y prevenir. La medicina alternativa parece ser producto de una fusión con la cultura industrial-mercantilista, cultura dominante en este 'mundo global'.

\section{¿Medicina complementaria basada en evidencias?}

La investigación biomédica en medicina complementaria está creciendo. El número de ensayos aleatorizados se duplica cada quinquenio $\left({ }^{28}\right)$. La conocida base de datos Cochrane Library le ha dedicado hasta la actualidad cerca de cincuenta revisiones sistemáticas $\left(^{8}\right)$.

En el caso de la homeopatía, uno de los metaanálisis más recientes analizó 186 estudios, la mayoría de ellos, estudios aleatorios a doble ciego, controlados por placebo $\left({ }^{29}\right)$. La revisión más reciente sobre tratamientos naturales (herbales) cita más de 100 publicaciones $\left({ }^{30}\right)$, entre ellas varios metaanálisis, uno de ellos sobre la famosa St. John's Wort (Hypericum perforatum) $\left({ }^{31,32}\right)$, otros dos sobre el uso del extracto de Ginkgo biloba $\left({ }^{33,34}\right)$. Adicionalmente, se cita decenas de revisiones sistemáticas. La medicina tradicional china también cuenta con estudios clínicos aleatorizados; una reciente revisión sistemática contabilizó más de $\left.7000{ }^{(35}\right)$.

Respecto a los productos naturales 'nacionales', su notoriedad parece ser solo en el ámbito peruano y regional. La uncaria tomentosa, por ejemplo, no es nombrada en la revisión sobre productos herbales referida anteriormente $\left({ }^{30}\right)$. Por otro lado, en la base de datos Medline ${ }^{36}$ ) solo se puede 'ubicar' cuatro revisiones sobre uña de gato; tres están publicadas en revistas de medicina alternativa; son revisiones acerca de los efectos inmunomoduladores del uncaria tomentosa $\left({ }^{37-39}\right)$. Una cuarta revisión hace referencia a la existencia de variantes quimotípicas de la planta $\left({ }^{40}\right)$. Hasta la actualidad, no se ha publicado algún estudio clínico aleatorio, hecho reconocido por una de la revisiones $\left({ }^{37}\right)$.

Otra hierba nacional con considerable información, que sin embargo no llega a traducirse en publicaciones internacionales, 
es la Croton lechleri, la popular 'sangre de grado'. Su resina, ampliamente utilizada, ha sido estudiada clínicamente solo en dos ensayos aleatorios controlados $\left({ }^{41,42}\right)$; hasta la actualidad, solo existe una revisión $\left({ }^{43}\right)$.

Respecto a otras hierbas, los estudios nacionales -aunque se cuentan por decenasno son publicados a texto completo en revistas científicas nacionales o extranjeras. Solo se ha publicado resúmenes, muchos de ellos en los congresos locales que anualmente se realizan $\left({ }^{44}\right)$. No existen estudios registrados en Medline.

La recientemente muy publicitada Morinda citrifolia, comercializada como el jugo $N o n i^{\circledR}$, cuenta con más de cien trabajos publicados; sin embargo, en dos revisiones $\left({ }^{45-46}\right.$ ) (una con más de 130 referencias y otra con 37) no se cita algún estudio clínico o aleatorio.

Por último, la base de datos Cochrane registra en total casi 4000 estudios referidos a la medicina alternativa $\left({ }^{8}\right)$.

\section{Razones teóricas de la incompatibilidad entre la medicina occidental y la alternativa}

Tradicionalmente, se considera a la inducción como aquella inferencia que, partiendo de premisas que describen hechos singulares o particulares, obtiene una conclusión de carácter universal. Se dice que la inducción nos permite generalizar una serie de observaciones para obtener una regla general, una ley. A primera vista, parece claro; sin embargo, el problema surge cuando preguntamos por qué debemos de aceptar que solo investigando unos pocos elementos podemos suponer conocer los demás elementos del universo.

Una manera de plantear solución al problema consistió en apelar a conceptos probabilísticos, según lo cual la inducción sería una inferencia no conclusiva ni definitiva, sino probable. Aparentemente, una hipótesis queda verificada cuando encontramos pruebas en la experiencia a favor de ella. Se propone que, cuanto más verificada es una hipótesis, es decir, cuanto mayor número de sucesos de verificación sucedan a favor de ella, la hipótesis es más válida. Sin embargo, como no podemos realizar todas las verificaciones posibles, ya que nuestro mundo es infinito al menos en un sentido (el tiempo), se propone que la hipótesis verificada o confirmada solo puede ser 'probable'.

Aunque eso parezca intuitivamente lógico y cierto, el filósofo Karl Popper se encargó de demostrarnos que tal razonamiento está en realidad errado. Aunque parezca paradójico decirlo, los científicos no buscarían las hipótesis más probables. Karl Popper se da cuenta que el intento de 'demostrar' una hipótesis en función de la confirmación por casos y explicar esta demostración en términos probabilísticos, no es posible. Afirma que, al aceptar el planteamiento del problema en forma probabilística, tendríamos primero que satisfacer los axiomas y el concepto mismo de probabilidad.

Si aceptáramos la probabilidad de un hecho, como el número de veces que ocurra sobre el total de sucesos o elementos en una secuencia determinada, aceptaríamos la demostración de una teoría (T) dependiendo del número de confirmaciones (E); esto es: $P(T / E)=n(t) / n(E)$. Dado que E puede ser infinito o al menos medido en eones, el número de secuencias posibles para $E$ es semejante al infinito. Por tanto, si reemplazáramos, tendríamos: $\mathrm{P}(\mathrm{T} / \mathrm{E})=0\left({ }^{47}\right)$. Mostrándose que la probabilidad de confirmar la teoría $\mathrm{T}$ por medio del número de confirmaciones empíricas puede ser cero o al menos cercano a cero, lo cual seria paradójico, ya que nos llevaría a concluir que no es posible generar leyes o enunciados universales.

Popper concluye que la confirmación de una teoría desde el punto de vista de pro- 
babilidades no es posible, ya que esta confirmación no es una función regular de aque1la. Las leyes universales y las teorías científicas simplemente no son verificables o confirmables, "es demasiado lo que afirman acerca del mundo, más de lo que podríamos 'verificar' o 'confirmar'" $\left({ }^{47}\right)$.

Lo anterior nos lleva a descubrir que es fácil obtener confirmaciones o verificaciones para casi cualquier teoría, proposición o hipótesis, si son confirmaciones las que buscamos. En contraposición a ello, Popper propone que las teorías científicas, para considerarlas como tales, deben ser refutables. La solución propuesta por Popper es, entonces, la de introducir el criterio de 'refutabilidad': "Las confirmaciones solo cuentan si son el resultado de predicciones riesgosas que, de no basarnos en la teoría en cuestión, habríamos esperado que se produjera un suceso que es incompatible con la teoría, un suceso que refutara la teoría” $\left({ }^{47}\right)$.

Así, toda 'buena' teoría debería implicar una prohibición. Esta prohibición debería estar expresada en las hipótesis de más bajo nivel observacional, a través de las cuales ponemos a prueba -o mejor dicho a constrastación- una teoría. Si la teoría aprueba con éxito, todo prueba genuina que busque refutarla o desmentirla, creemos más en ella. Pero, si la observación muestra que el efecto o hecho predicho por la hipótesis (o las hipótesis) de más bajo nivel observacional se encuentran ausentes, entonces la teoría queda simplemente refutada.

En añadidura, la ciencia se sirve de datos teóricos y de otras teorías para proponer una explicación, una descripción, o una solución a las observaciones iniciales; propuesta que tiene carácter de verdad, siempre y cuando resista a la refutación. Aquí debemos de agregar la aclaración que hace Mario Bunge: no solo contrastamos nuestras hipótesis o teorías haciendo uso de la refutación o 'contrastación' empírica, sino, además, haciendo uso de la 'contrastación' teórica.

Esto nos lleva a comparar el fondo teórico de las medicinas alternativas, fondo teórico que es muy distinto al que acepta la ciencia. En el caso específico de las ciencias biológicas, y por extensión de toda técnica que se fundamenta en ellas, su fondo teórico asume una ontología francamente naturalista, naturalista porque se ocupa de organismos, no de entes etéreos sin materia conocida. Otro componente es su metodología; la 'medicina occidental' asume el método científico al realizar sus investigaciones; este método ayuda en su cometido de encontrar leyes, hechos comunes, generales, claramente extrapolables; por ello, desconfiamos de la anécdota, el relato o el 'testimonio' (muy usado en la medicina tradicional).

Si asumimos todo lo anterior, debemos inferir, entonces, que las medicinas alternativas (desde las medicinas tradicionales, folklóricas, hasta la homeopatía) no son en su conjunto compatibles con el pensamiento científico.

Por ejemplo, la homeopatía tiene entre sus principios terapéuticos el de la similitud y el uso de las diluciones llamadas 'potencias' $\left({ }^{48}\right)$. Según el primero, los pacientes con una característica particular de síntomas y signos pueden ser curados si se les proporciona una terapia que produzca los mismos síntomas en un individuo saludable. El segundo principio propone que, los remedios homeopáticos retienen actividad biológica si aquellos son repetidamente diluidos y agitados; se dice, inclusive, que el líquido debe ser diluido más allá del número de avogrado (algo similar a diluir cualquier tableta conocida en el océano Pacífico o Atlántico). La homeopatía dice que esta solución 'retendría' la información de la sustancia originalmente diluida; gracias a ese recuerdo; esta solución posee 'propiedades' terapéuticas $\left({ }^{49}\right)$. 
Si preguntamos a cualquier farmacólogo o bioquímico aplicado, éste nos dirá que al cabo de las numerosas diluciones sucesivas involucradas en la fabricación de un fármaco, no existirán suficientes moléculas, digamos por centímetro cúbico de diluyente, como para que esta molécula tenga posibilidades de ejercer alguna interacción, peor aún algún efecto. El agua, en estado líquido, no tiene estructura suficiente para recordar lo que contuvo en el pasado.

Otro ejemplo, es el caso de la acupuntura. De acuerdo con la tradicional medicina china $\left({ }^{50}\right)$, el funcionamiento del cuerpo humano está controlado por una fuerza o energía vital, llamada 'Qi'; esta energía circularía entre los órganos a lo largo de canales de energía, llamados meridianos. Según esta doctrina 'existen' doce meridianos principales, los cuales corresponden a 'las doce funciones principales' del cuerpo humano. La energía 'Qi' debe fluir siempre en cantidad y calidad adecuadas a través de los meridianos, para asegurar buena salud. Los puntos de acupuntura estarían localizados a lo largo de los meridianos, convirtiéndose en medios físicos para alterar el flujo del 'Qi'. El tratamiento, colocando agujas o administrando presión sobre tales puntos, tendría como misión regular el flujo de energía. Un dato curioso adicional es que los puntos de acupuntura pueden variar según las técnicas. Existirían diferencias de localización de los puntos entre las técnicas chinas, japonesas y occidentales. La técnica coreana reporta, por ejemplo, hasta 150 puntos $\left({ }^{51}\right)$. En ningún caso, se ha podido 'ubicar' físicamente (ya sea a nivel molecular, eléctrico o electromagnético) las relaciones de energía entre los puntos de acupuntura, los meridianos y los órganos o 'las funciones vitales'.

Si consideramos a la verdad como la relación biunívoca entre lo que se dice y lo que 'es', no podemos aceptar 'diferentes verdades'; de las diferentes interpretaciones a un hecho solo una puede ser cierta; en el caso de la terapéutica mediante la administración de una sustancia, cualquiera sea ésta, es un hecho universal que esta sustancia debe además de existir ser capaz de interactuar con la materia (ya sea a nivel molecular, eléctrico, electromagnético o cualquier otra interacción fisicoquímica); si no aceptamos esto, estamos negando los millares de estudios realizados para conocer y demostrar la eficacia de los fármacos que usamos.

Pero, entonces ¿qué pasa con los estudios sobre tratamientos homeopáticos que, como vimos anteriormente, muestran mayor efecto que placebo? $\left({ }^{29}\right)$. La explicación estaria en la tesis de Popper, sobre la inducción. La confirmación no basta; al parecer esto no es muy consciente en algunos empiristas ingenuos, quienes piensan que basta con realizar estudios de investigación sobre los tratamientos alternativos para verificar su eficacia. Lo más importante es refutarlos, en el caso más sencillo ejecutar los mismos estudios, bajo las mismas condiciones que han obtenido mayor efecto que placebo, hecho que no existe. Por ejemplo en el metaanálisis referido anteriormente no existe en caso alguno un estudio homeopático que haya sido repetido por al menos tres investigadores independientes.

En el caso de la acupuntura, dos revisiones sistemáticas recientes $\left({ }^{50}\right)$ indican que existirían solo dos aplicaciones terapéuticas, donde los estudios confirman mayor eficacia terapéutica de la acupuntura contra el placebo; estos son, el dolor dental postoperatorio y las náuseas y vómitos posquimioterapia. En otras indicaciones, clamadas por los acupunturitas, los estudios son contradictorios, mostrando algunos estudios mayor efecto contra placebo y otros iguales o incluso menor efecto que el placebo. Otro aspecto a señalar es que la mayoría de las condiciones que los acunpunturistas tratan y donde existen algunos estudios a su favor son patologías autolimitadas, de cursos remitentes y donde siempre existe involucrado un componente subjetivo ${ }^{50-52}$. 
Además de las fallas técnicas, los estudios en medicina alternativa no han sido refutados en el sentido popperiano.

En el campo de la etnofarmacología, específicamente la medicina herbal, algunos claman que validar científicamente el uso de ciertos productos derivados de plantas significa validar el 'constructo' medicina tradicional; es decir, significaría validar el conjunto de creencias populares respecto a las plantas medicinales. Sin embargo, se debe mencionar que al comprobar científicamente la eficacia de algunos componentes de las plantas medicinales y luego de ello usarlas, no estamos haciendo medicina tradicional ni folclórica; todo lo contrario, estamos entregándonos a la 'medicina occidental'. El uso tradicional (etnofarmacológico) de estas plantas sirve como un indicio de eficacia; nos sugiere una posibilidad terapéutica, mas no la refrenda. Se debe recordar que las pruebas de eficacia no son solo de nivel bioquímico o fisiológico (es decir, de explicación causal), sino fundamentalmente de orden clínico. A la medicina científica no le basta que un determinado fármaco cause algún efecto bioquímico en las células o en las moléculas de probeta, sino fundamentalmente que el efecto se traduzca en el humano vivo y enfermo. Por ello, son los estudios aleatorizados a doble ciego los que mejor 'demuestran' la eficacia de un fármaco; este debe vencer muchas variables para que sea clínicamente efectivo.

Las explicaciones mágicas y míticas de las medicinas tradicionales tienen menos oportunidad de ser verdaderas, ya que su andamiaje no comprende una ontología naturalista (puesto que acude a entes inmateriales: almas, espíritus, canales de energía y conexiones no objetivables). Su conocimiento ha sido logrado a partir de la experiencia, pero la experiencia no controlada ni medida; la contrastación de sus hipótesis no les preocupa demasiado. Por ello, quizá esta medicina sigue siendo milenaria, a pesar de aventajar en miles de años a la medicina científica, la medicina tradicional se ha mantenido casi estática, sin cambios. Esto prueba que la herramienta cognoscitiva que utilicemos, así como el andamiaje teórico que asumamos, es lo que verdaderamente nos posibilita el conocer. Como dijéramos en la parte introductoria, la medicina occidental no posee ni dos siglos; sin embargo, nuestro conocimiento -aunque aún limitado- ha avanzado extraordinariamente. La razón: aceptar y utilizar la ciencia.

Algunos reclaman que esto no debe interesarnos, ya que, al ser la ciencia una construcción social, la verdad (el conocimiento) sería relativa y por ello dos teorías provenientes de 'sociedades' distintas pueden ser válidas. Por tanto, a la medicina china no le debe importar ser 'coherente' con el supuesto conocimiento occidental. Quienes abrazan esta posición aceptan el relativismo epistemológico, doctrina según la cual toda verdad es relativa al sujeto, grupo social o periodo histórico. En otras palabras, no habría verdades objetivas universales. Empero, el conocimiento científico es diferente. La ciencia es universal; la ciencia no solo se vale de opiniones sino de hechos contrastables. Si una opinión solo vale para los miembros de algún grupo social, entonces es ideológica o estética, no científica ${ }^{(53)}$. Por último, si el relativismo fuera verdadero, sería entonces falso, ya que no es sino un producto efímero de un grupo social transitorio. Quizás entonces todavía podemos confiar en la posibilidad de alcanzar la verdad y gracias a ella aliviar a aún más sanar.

\section{REFERENCIAS BIBLIOGRÁFICAS}

1. Sigerist H, Aguirre R. (trad.) Civilización y Enfermedad. México: Fondo de Cultura Económica; 1946.

2. Flexner A. Medical education in the United States and Canada (Bulletin No. 4). New York: Carnegie Foundation for the Advancement of Teaching Boston, MA: Updyke; 1910.

3. Regan-Smith M. Reform without change: update, 1998. Acad Med. 1998;73:505-7. 
4. Eisenberg DM, Kessler RC, Foster C, Norlock FE, Calkins DR, Delbanco TL. Unconventional medicine in the United States - prevalence, costs, and patterns of use. N Engl J Med. 1993;328:246-52.

5. Vandenbroucke JP, de Craen AJ. Alternative medicine: a «mirror image» for scientific reasoning in conventional medicine. Ann Intern Med. 2001;135:507-13.

6. Maguiña C. La medicina científica occidental, otras alternativas y las plantas medicinales: Una nueva visión. Diagnóstico. 2002;41.

7. Zollman C, Vickers A. ABC of complementary medicine: What is complementary medicine? BMJ. 1999;319:693-6.

8. The Cochrane Collaboration [sede Web]. New Jersey: John Wiley \& Sons; c2000-2007 [citado el 16 agosto de 2002]. Disponible en: http://www.cochrane.org/

9. Organización Mundial de la Salud. Estrategia de la OMS Sobre Medicina Tradicional 2002-2005. Ginebra: Organización Mundial de la Salud; 2002 (Documento de referencia WHO/EDM/TRM 2002.1).

10. Angell M, Kassirer J. Alternative medicine. The risks of untested and unregulated remedies. N Engl J Med. 1998;339:839-41.

11. Cooper RA, Stoflet SJ. Trends in the education and practice of alternative medicine clinicians. Health Aff (Millwood). 1996;15:226-38.

12. Spiegel D, Stroud P, Fyfe A. Complementary medicine. West J Med. 1998;168:241-7.

13. Pelletier KR, Marie A, Krasner M, Haskell WL. Current trends in the integration and reimbursement of complementary and alternative medicine by managed care, insurance carriers, and hospital providers. Am J Health Promot. 1997;12:112-22.

14. Universidad San Martin de Porres [sede Web]. Lima: Facultad de Medicina, Universidad San Martin de Porres [citado el 6 junio de 2006]. V Congreso Mundial de Medicina Tradicional 2005. Disponible en: http:// www.medicina.usmp.edu.pe/congresomundial/

15. Essalud [sede Web]. Lima: Essalud [citado el 16 de agosto de 2002]. Programa de medicina complementaria 2002. Disponible en: http://www.essalud.sld.pe/iesp/medcomp.htm

16. Leibovici L. Alternative (complementary) medicine: a cuckoo in the nest of empiricist reed warblers. BMJ. 1999;319:162932 .

17. Mason S, Tovey P, Long F. Evaluating complementary medicine: methodological challenges of randomised controlled trials. BMJ. 2002;325:832-34.

18. Heidelberg A. News extra: Three out of four Germans have used complementary or natural remedies. BMJ. 2002;325:990.

19. Organización Mundial de la Salud. Complementary and Alternative Medicines and Therapies. Washington, DC: Oficina Regional de la OMS para las Américas/Organización Panamericana de la Salud (grupo de trabajo OPS/OMS); 1999.

20. Valdivia F, Hidalgo M. Uso de la medicina tradicional en diabetes mellitus no insulino-dependiente. An Fac Med Lima. 1996;57:180-3
21. Arroyo J. El proceso del sector salud 1990-1995: colapso operativo y reforma sanitaria silenciosa. An Fac Med Lima. 1996;57:188-95.

22. Schechter A. The crisis in clinical research: Endangering the half-century National Institutes of Health Consensus. JAMA. 1998;280:1440-2.

23. National Center for Complementary and Alternative Medicine [sede Web]. Bethesda: NCCAM [citado el 14 de diciembre de 2002]. Five year strategic plan 2001. Disponible en: http:/ /nccam.nih.gov/

24. Marwick C. Alterations are ahead at the OAM. JAMA 1998;280:1553-4.

25. Lewith G. Complementary medicine must be research led and evidence based. BMJ. 2000;320:188.

26. Ernst E. Regulating complementary medicine. BMJ. 1996;313:882.

27. Eisenberg DM, Davis RB, Ettner SL, Appel S, Wilkey S, Van Rompay M, Kessler RC. Trends in alternative medicine use in the United States, 1990-1997: results of a follow-up national survey. JAMA. 1998;280:1569-75.

28. Vickers AJ. Bibliometric analysis of randomised controlled trials in complementary medicine. Complementary Theor Med. 1998;6:185-9.

29. Linde K, Clausius N, Ramirez G, Melchart D, Eitel F, Hedges LV, et al. Are the clinical effects of homeopathy placebo effects? A meta-analysis of placebocontrolled trials. Lancet. 1997;350:834-43.

30. De Smet P. Herbal remedies. N Engl J Med. 2002;347:2046-56.

31. Williams JW Jr, Mulrow CD, Chiquette E, Noel PH, Aguilar C, Cornell J. A systematic review of newer pharmacotherapies for depression in adults: evidence report summary. Ann Intern Med. 2000;132:743-56.

32. Whiskey E, Werneke U, Taylor D. A systematic review and meta-analysis of Hypericum perforatum in depression: a comprehensive clinical review. Int Clin Psychopharmacol. 2001;16:239-52.

33. Pittler MH, Ernst E. Ginkgo biloba extract for the treatment of intermittent claudication: a meta-analysis of randomized trials. Am J Med. 2000;108:276-81.

34. Moher D, Pham B, Ausejo M, Saenz A, Hood S, Barber GG. Pharmacological management of intermittent claudication: a meta-analysis of randomised trials. Drugs. 2000;59:105770.

35. Tang J, Zhan S, Ernst E. Review of randomised controlled trials of traditional Chinese medicine. BMJ. 1999;319:1601.

36. National Library of Medicine [sede Web]. Bethesda: NLM [citado el 22 de junio de 2003]. Disponible en: http:// www.ncbi.nlm.nih.gov

37. Williams JE. Review of antiviral and immunomodulating properties of plants of the Peruvian rainforest with a particular emphasis on una de gato and sangre de grado. Altern Med Rev. 2001;6:567-79.

38. Keplinger K, Laus G, Wurm M, Dierich MP, Teppner H. Uncaria tomentosa (Willd.) DC.--ethnomedicinal use and 
new pharmacological, toxicological and botanical results. J Ethnopharmacol. 1999;64:23-34.

39. Syrimis A. Uncaria tomentosa: a review. British J Phytotherapy. 1998;5:29-31.

40. Reinhard KH. Uncaria tomentosa (Willd.) D.C.: cat's claw, una de gato, or saventaro. J Altern Complement Med. 1999;5:143-51

41. Holodniy M, Koch J, Mistal M, Schmidt JM, Khandwala A, Pennington JE, et al. A double blind, randomized, placebo-controlled Phase II study to assess the safety and efficacy of orally administered SP-303 for the symptomatic treatment of diarrhea in patients with AIDS. Am J Gastroenterol. 1999;94:3267-73.

42. Orozco-Topete R, Sierra-Madero J, Cano-Dominguez C, Kershenovich J, Ortiz-Pedroza G, Vazquez-Valls E, et al. Safety and efficacy of Virend for topical treatment of genital and anal herpes simplex lesions in patients with AIDS. Antiviral Res. 1997;35:91-103.

43. Meza EN, ed. Desarrollando nuestra diversidad biocultural: «Sangre de grado» y el reto de su producción sustentable en el Perú. Lima: Universidad Nacional Mayor de San Marcos; 1999.

44. Libro de Resúmenes del Segundo Curso International de Plantas Medicinales y Fitoterapia. Lima: Instituto de Fitoterapia Americano, 2002.

45. Wang MY, West BJ, Jensen CJ, Nowicki D, Su C, Palu AK, et al. Morinda citrifolia (Noni): A literature review and recent advances in Noni research. G Acta Pharmacol Sin. 2002;23(12):1127-41.

46. McClatchey W. From Polynesian healers to health food stores: Changing perspectives of Morinda citrifolia
(Rubiaceae). Integrative Cancer Therapies. 2002;1:110-20.

47. Popper KR, Sánchez de Zavala (trad.) La Lógica de la Investigación Científica. Madrid: Tecnos; 1962. Originalmente publicado, en inglés: The Logic of Scientific Discovery. London: Hutchinson; 1959.

48. Hahnemann S. Organon of medicine. Los Angeles: JP Tarcher; 1982.

49. Endler PC, Schulte J. Ultra high dilution: physiology and physics. Dordrecht: Kluwer; 1994.

50. Breuner C. Alternative and complementary therapies. Adolescent Medicine Clinics. 2006;17:521-46.

51. Kim YS, Jun H, Chae Y, Park HJ, Kim BH, Chang IM, et al. The practice of Korean medicine: an overview of clinical trials in acupuncture. Evid Based Complement Alternat Med. 2005;2:325-52.

52. Carpenter J, Neal J. Other complementary and alternative medicine modalities: acupuncture, magnets, reflexology, and homeopathy. Am J Med. 2005;118:109S-17S.

53. Bunge M. Sistemas Sociales y Filosofía. Buenos Aires: Editorial Sudamericana; 1995. p. 182-4.

Manuscrito recibido el 20 de enero de 2007 y aceptado para publicación el 22 de febrero de 2007.

Correspondencia

Adolfo Pena, MD

Good Samaritan Hospital

375 Dixmyth Ave.

Cincinnati, OH 45220 USA

Correo-e: adolfo_pena@trihealth.com 